\title{
Dissection of the Endolymphatic Sac from Mice
}

\author{
Keiji Honda ${ }^{*}, 1$, , Hyun Jae Lee ${ }^{*}, 1$, Andrew J. Griffith ${ }^{1,3}$, Isabelle Roux ${ }^{1}$ \\ ${ }^{1}$ Otolaryngology Branch, National Institute on Deafness and Other Communication Disorders, National Institutes of Health ${ }^{2}$ Department of \\ Otorhinolaryngology, Tokyo Medical and Dental University ${ }^{3}$ Department of Otolaryngology, College of Medicine, University of Tennessee Health Science \\ Center \\ *These authors contributed equally
}

\section{Corresponding Authors}

Keiji Honda

honda.oto@tmd.ac.jp

Isabelle Roux

isabelle.roux@nih.gov

\section{Citation}

Honda, K., Lee, H.J., Griffith, A.J.,

Roux, I. Dissection of the Endolymphatic

Sac from Mice. J. Vis. Exp. (169),

e62375, doi:10.3791/62375 (2021).

\section{Date Published}

March 29, 2021

DOI

$10.3791 / 62375$

URL

jove.com/video/62375

\section{Abstract}

The study of mutant mouse models of human hearing and balance disorders has unraveled many structural and functional changes which may contribute to the human phenotypes. Although important progress has been done in the understanding of the development and function of the neurosensory epithelia of the cochlea and vestibula, limited knowledge is available regarding the development, cellular composition, molecular pathways and functional characteristics of the endolymphatic sac. This is, in large part, due to the difficulty of visualizing and microdissecting this tissue, which is an epithelium comprised of only one cell layer. The study presented here describes an approach to access and microdissect the endolymphatic sac from the wild-type mouse inner ear at different ages. The result of a similar dissection is shown in a pendrin-deficient mouse model of enlargement of the vestibular aqueduct. A transgenic mouse with a fluorescent endolymphatic sac is presented. This reporter mouse can be used to readily visualize the endolymphatic sac with limited dissection and determine its size. It can also be used as an educational tool to teach how to dissect the endolymphatic sac. These dissection procedures should facilitate further characterization of this understudied part of the inner ear.

\section{Introduction}

The mammalian inner ear consists of the cochlea, the saccule, the utricle, three semi-circular canals, and the endolymphatic sac (Figure 1A). These organs comprise a continuous, fluid-filled epithelium called the membranous labyrinth, with adjacent organs connecting directly or through small canal structures such as the ductus reuniens, saccular duct, utricular duct, or endolymphatic duct. Compared to other organs of the inner ear, the endolymphatic sac has unique characteristics. First, it lacks sensory epithelial cells. Instead, the endolymphatic sac has cells specialized for ion transport. Second, although the membranous labyrinth is enclosed in the bony labyrinth, the endolymphatic sac is an exception, protruding partially out of the petrous bone and into the intracranial cavity. This morphology appears to be 
highly conserved during evolution of the inner ear. Third, the endolymphatic sac is the first structure to develop from the otocyst at an early embryonic stage prior to formation of other organs. In addition several pathologies have been associated with an enlarged endolymphatic sac or an abnormal endolymphatic compartment. The presence of pathogenic variants in SLC26A4 (also known as the pendrin gene) leads to a comparatively common form of sensorineural hearing loss, caused by the enlargement of the endolymphatic sac associated with the presence of an enlarged vestibular aqueduct $(E V A)^{1}$. When associated with goiter, it is refered to as Pendred syndrome ${ }^{2,3}$. Meniere's disease is also thought by some to be associated with abnormality of the endolymphatic compartment (hydrops) ${ }^{4}$. The unique features of the endolymphatic sac and pathologies associated with a change of its morphology are consistent with a critical role for development and maintenance of the inner ear.

Despite its importance, knowledge is still limited regarding the development, cellular composition, molecular pathways and functional characteristics of the endolymphatic sac. This is at least partly due to the difficulty of visualizing and microdissecting this tissue. The endolymphatic sac is a pouch-shaped structure, composed of a single layer of epithelial cells, which is often translucent and very difficult to identify out of the conjunctive tissue which surrounds it by light stereomicroscopy. Although a small number of researchers have developed whole-mount dissection techniques and published their experimental findings $5,6,7,8,9,10$, there is no publication focusing on the technical details of this procedure.

In this study, the microdissection approaches developed to access and isolate the endolymphatic sac from the wild-type mouse inner ear at different ages, in wholemount, is described. The results of a similar dissection is shown in a mouse model, lacking expression of SLC26A4, which has enlargement of the endolymphatic duct and sac. A mouse line with a transgene encoding a Cre recombinase expressed in the endolymphatic sac is reported. In presence of a fluorescent reporter of Cre expression, here tdTomato, the fluorescence can be used to readily visualize the endolymphatic sac with limited dissection and to obtain information about its size. This reporter mouse line can also be used as an educational tool to practice and perfect endolymphatic sac dissections. The ability to dissect endolymphatic sac tissue should facilitate further characterization of this understudied but essential component of the inner ear.

\section{Protocol}

All animal experiments and procedures were performed according to protocols approved by the Animal Care and Use Committees of the National Institute of Neurological Diseases and Stroke/National Institute on Deafness and Other Communication Disorders (\#1264) and the Institutional Animal Care and Use Committee at Tokyo Medical and Dental University (A2020-058A).

NOTE: C57BL/6J mice (stock number no. 000664), Pds ${ }^{-/}$also known as S/c26a4 $4^{-/-}$mice (129S-S/c26a4tm1Egr/AjgJ, no. 018424) ${ }^{\text {11, B }}$, B6:CBA$\mathrm{Tg}$ (ATP6V1B1-Cre) ${ }^{1 \mathrm{Rnel} / \mathrm{Mm}}$ mice $^{12}$ and Ai9(LSL-RCL-tdT) (B6.Cg-Gt(ROSA)26Sor tm9(CAG-tdTomato)Hze, no. 007909) mice $^{13}$ are available from the Jackson Laboratory and upon request. All genotyping procedures have previously been described. B6;CBA-Tg(ATP6V1B1-Cre) $)^{1 \text { Rnel/Mm mice }}$ previously backcrossed to strain $\mathrm{C} 57 \mathrm{BL} / 6 \mathrm{~J}$ for at least five generations, were bred for experiments. Animals of both sexes can be studied with this approach without an obvious 
difference. The first day after overnight mating is counted as embryonic day 0.5 (E0.5).

\section{Harvest and fixation of the intact inner ear in the skull}

1. Euthanize experimental animal or time-pregnant dam using an institutionally approved procedure, for example, using $\mathrm{CO}_{2}$ inhalation. Work under a fume hood if possible, to avoid or minimize the spread of mouse allergens. After verifying the lack of response to painful stimuli such as paw or tail pinches, decapitate the animal. For a time-pregnant dam, cervical dislocation can be used as an alternative to decapitation if its inner ears are not used for further experiments.

2. Harvest the embryos by laparotomy ${ }^{14}$ using artery scissors with a ball tip to protect the embryos during the process. Manipulate the embryos using ring forceps. Place the uterine horns in $4{ }^{\circ} \mathrm{C}$ PBS on ice and isolate each embryo as previously described ${ }^{15}$ before decapitating them.

3. Bisect the head from back to front: first incise the top of the head by first incising the skin, then the top of the skull, incising about one third of it with each cut so there is no bending of the skull. Then cut the bottom of the skull at once.

NOTE: For embryos, the use of a sharp blade, rather than scissors, is advised. It is critical that, at all ages, the skull is not deformed while bisecting it.

4. Without touching or bending the skull, carefully remove the hemi-brain to reveal the bony labyrinth (Figure 1B,

\section{Supplementary Figure 1A-B).}

5. For mice postnatal day 5 or older, transect the external auditory canal and pull the skin forward towards the nose.
Trim the skull around the inner ear (Supplementary Figure 1C).

6. Transfer the two hemi-skulls of each animal with the inner ear, as intact as possible, to a glass vial or tube containing $10 \mathrm{~mL}$ of $4{ }^{\circ} \mathrm{C} 4 \%$ paraformaldehyde (PFA) diluted in 1X PBS and keep on ice under a fume hood.

NOTE: Do not try to remove the inner ear from the skull at this step as this may damage the endolymphatic duct and sac while doing so.

7. Incubate for $1 \mathrm{~h}$ at $4{ }^{\circ} \mathrm{C}$ on a rocking shaker. If working with tissue from an animal with a fluorescent reporter, maintain the tube containing the specimen in aluminum foil or in a box to minimize exposure to light.

NOTE: If interested in studying microtubules and associated molecules, tissue fixation should be performed at room temperature (RT) with RT fixative.

8. Discard the PFA in an appropriate container in a fume hood. Wash three times with $10 \mathrm{~mL}$ of $1 x$ PBS for 15 min each time on a rocking shaker at $\mathrm{RT}$ to eliminate the fixative before dissection.

\section{Endolymphatic sac microdissection}

1. Transfer each inner ear preparation to a $35 \mathrm{~mm}$ tissue culture dish containing 1x PBS.

2. Place the preparation in the dish so that the root of the 8th cranial nerve is oriented up. Hold the tissue in this position by grasping the cochlea with a \#4 forceps (Figure 1C).

3. Identify important landmarks: the vestibular aqueduct, the anterior and posterior semicircular canals, common crus, and sigmoid sinus (vein) (Figure 1D).

NOTE: The endolymphatic sac in the wild type mouse is translucent and hard to visualize. For successful 
microdissection of the endolymphatic sac, it is important to understand its anatomy and localization (Figure 1E).

4. Incise the dura mater and vestibular aqueduct, as well as the underlying connective tissues surrounding the endolymphatic sac, using a $27 \mathrm{G}$ needle on a $1 \mathrm{~mL}$ syringe (Figure 1D,F).

NOTE: For an adult mouse, decalcification of the preparation using 10\% EDTA $(\mathrm{pH} \quad 7.4)$ before microdissection is useful, but not essential, to facilitate incision of the vestibular aqueduct. For dissection of opened endolymphatic sac older than postnatal day 5 , it is recommended to position the incision line on the vestibular aqueduct slightly anterior to make an incision into the endolymphatic sac lumen (Figure 2A).

5. Hold the connective tissue located lateral to the endolymphatic sac with forceps and pull up the tissue to peel it from the temporal bone (Figure 1G).

6. Carefully remove any remaining debris. The preparation usually includes the endolymphatic sac epithelium, surrounding connective tissues, a portion of the vestibular aqueduct, and the sigmoid sinus (Figure 1H-I). If necessary, separate the endolymphatic sac epithelium from surrounding tissues (Figure 1J).

7. (Optional) For dissection of the opened endolymphatic sac, hold the stem part of the preparation so that the cross-section of the lumen can be observed. Insert a $27 \mathrm{G}$ needle into the lumen and move it to cut the endolymphatic sac into two sheets (Figure 2B-C). Hold the edge of each sheet-like tissue with forceps and separate them from each other (Figure 2D-F).

\section{Immunohistochemistry}

1. After microdissection, use \#5 forceps to transfer endolymphatic sacs into a 9-well spot glass plate.

2. Using the microscope to make sure the endolymphatic sacs remain untouched, use a $200 \mu \mathrm{L}$ pipette to remove all 1x PBS except one drop containing the tissue. Add $200 \mu \mathrm{L}$ of permeabilizing and blocking solution (1x PBS with $0.15 \%$ Triton X-100 (PBS-TX) and $5 \%$ bovine serum albumin (BSA)) and incubate for $1 \mathrm{~h}$ at RT on an orbital shaker.

3. After removing all blocking solution except one drop containing the tissue, add $200 \mu \mathrm{L}$ of primary antibodies and incubate overnight at $4{ }^{\circ} \mathrm{C}$. Anti-pendrin antibodies ${ }^{6}$ diluted 1:1000 in blocking solution can be used to identify the mitochondria-rich cells of the endolymphatic sac, although a faint expression is also seen in a subset of ribosome-rich cells ${ }^{16}$ (Figure 5).

4. After three washes in $1 \times$ PBS for 15 min at RT, incubate with secondary antibodies. For the labeling presented here, incubate $200 \mu \mathrm{L}$ of fluorescent dye-conjugated secondary antibodies, diluted from the commercial stock at 1:500 in blocking solution, with the tissue on a rocking platform for $1 \mathrm{~h}$ at $\mathrm{RT}$ in the dark. Fluorescent dye-conjugated phalloidin, which recognizes $\beta$-actin (ACTB), can be used to highlight the presence of the endolymphatic sac and duct as well as the conjunctive tissue around them (Figure 5A, C, F-G).

5. Mount endolymphatic sacs between a glass slide and coverslip using an antifade mounting medium with DAPI. After letting the slides dry protected from light for $1 \mathrm{~h}$ at RT, apply transparent nail polish at the junction between coverslip and glass slide to seal them and limit air bubble 
formation. If trying to visualize the differential distribution of proteins at the apical versus the basolateral surface of the epithelial cells, it is recommended to open the sac during the mounting step by incising an edge and opening it.

6. Image using a confocal microscope. First visualize endolymphatic sac preparations with a $10 x$ objective to provide a global view of the sac labeling (Figure 5A-D). Then use a $63 x$ objective to gain a detailed view of the distribution of the protein of interest in mitochondria-and ribosome-rich cells of the endolymphatic sac (Figure 5FG).

\section{Representative Results}

Each step of this microdissection of a wild type endolymphatic sac from a postnatal day 5 (P5) mouse is detailed in the associated video and snapshots of the key steps of this dissection and opening of the endolymphatic sac are presented in Figure 1 and Figure 2.

Representative results of the dissections of the endolymphatic sac with tdTomato fluorescence at embryonic day 16.5 (E16.5), P5 and P30, following this protocol are presented in Figure 3 and in Supplementary Figure 1 and Supplementary Figure 2. Using a dissection microscope with fluorescence, the endolymphatic sac can be readily visualized in the skull after removal of the brain in mice carrying the transgene $\mathrm{Tg}(\mathrm{ATP} 6 \mathrm{~V} 1 \mathrm{~B} 1-\mathrm{Cre})$ in the presence of the reporter of Cre expression: Ai9 (LSL-RCL-tdT) (see Figure 3A-B, and Supplementary Figure 1 for images captured in incident light and tdTomato fluorescence of the endolymphatic sac at different stages of the dissection). In these mice, Cre is expressed as a mosaic in cells all along the endolymphatic sac and duct (Figure 3, see also Supplementary Figure $\mathbf{2}$ for visualization of the endolymphatic sac with versus without fluorescent reporter). Although the expression of this reporter is not specific for the endogenous expression of Atp6v1b1, which is restricted to the mitochondria-rich cells of the endolymphatic $\operatorname{sac}^{16}$, one of the two cell types of this epithelium (Figure 5E), it is a helpful aid to identify and dissect the endolymphatic sac.

Inner ears dissected from a mouse model for enlargement of the vestibular aqueduct are presented in Figure 4. As compared to control littermates, the endolymphatic sacs and ducts of mice deficient for SLC26A4 (pendrin) are enlarged.

Representative images of immunohistochemistry of intact endolymphatic sac with and without conjunctive tissue are shown in Figure 5. Results of immunohistochemistry of opened endolymphatic sac of a P5 wild type mouse are also presented (Figure 5F-G). The endolymphatic sac is composed of a single layer of epithelium folded in a pouch-shaped structure, containing in its lumen endolymph. This epithelium consists of two cell types, mitochondria-rich cells and ribosome-rich cells (Figure 5E). A whole-mount endolymphatic sac will be flattened because endolymph is no longer present. The sac will appear as two intertwined layers of epithelium, making it difficult to determine subcellular localization of proteins expressed in this epithelium. By opening the endolymphatic sac, the single layer epithelium can be readily visualized and the relative distribution of the proteins of interest, with respect to the endolymphatic sac lumen, can be determined more definitively. A potential apical (luminal, where endolymph would be) versus basal enrichment of these proteins can be more accurately determined. As an example, SLC26A4 is enriched on the apical side of mitochondria-rich cells (Figure $5 F)$. 

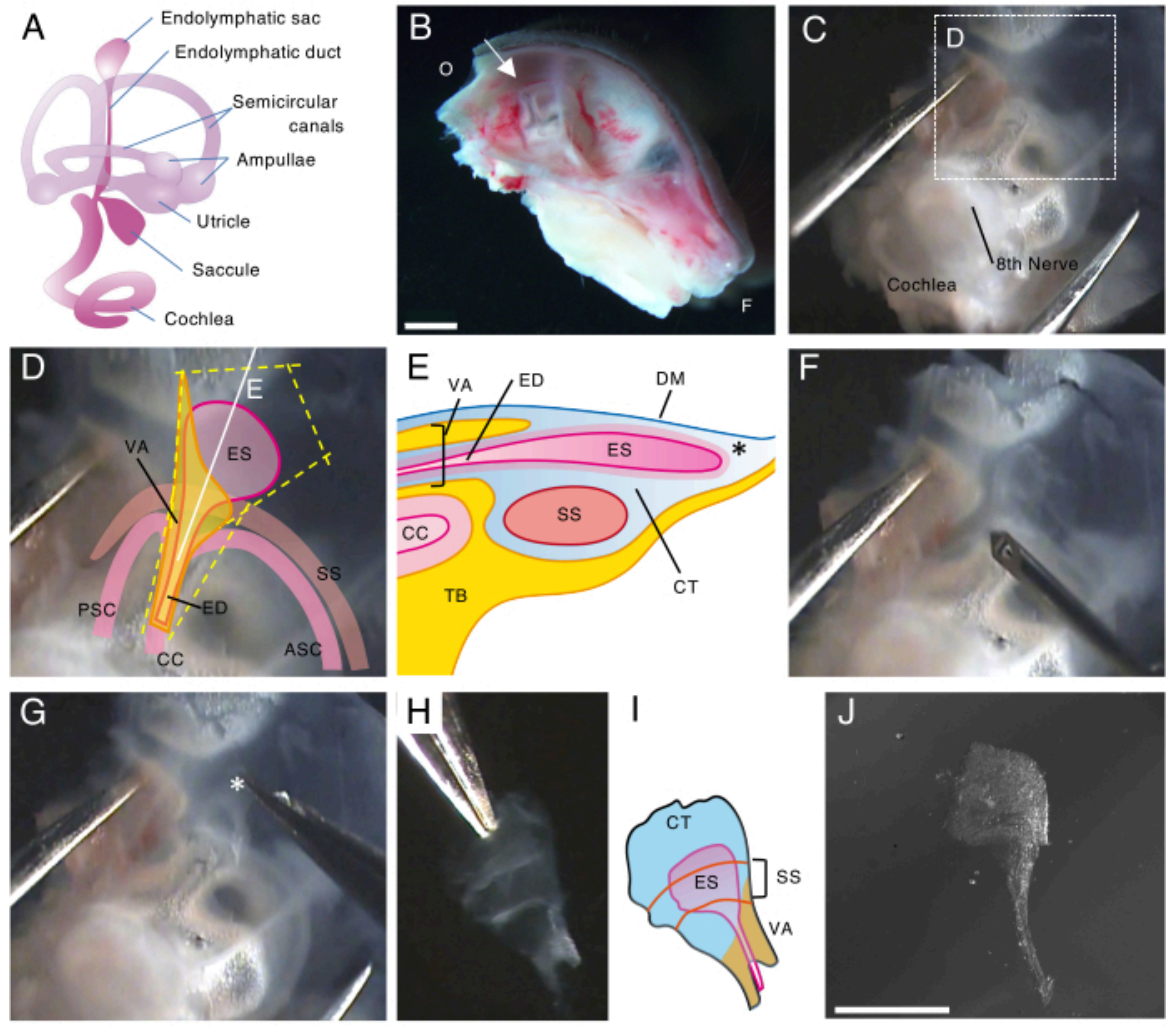

\section{Figure 1. Whole-mount dissection of the endolymphatic sac of a wild type mouse at post natal day 5 (P5).}

(A) Schematic of the membranous labyrinth of a developed mouse inner ear (right). The location of the endolymphatic sac, and the cochlea and vestibular structures are indicated. This figure has been modified from Honda et al. ${ }^{16}$.

(B) Mid-sagittal section showing the left hemi-skull. The location of the endolymphatic sac is indicated (arrow). (O, occipital;

$\mathrm{F}$, frontal)

(C) Otic capsule along with the squamous part of the temporal bone.

(D) Important landmarks overlaid onto the dotted box in panel C. (ES, endolymphatic sac; ED, endolymphatic duct; VA, vestibular aqueduct; ASC, anterior semicircular canal; PSC, posterior semicircular canal; CC, common crus; SS, sigmoid sinus)

(E) Schematic illustration of the cross-section along with the continuous white line on panel D through the endolymphatic sac. The proximal side of the endolymphatic sac is covered by a bony canal called the vestibular aqueduct, along with the endolymphatic duct. In the distal side, the endolymphatic sac, surrounded by conjonctive tissue (CT), extends and protrudes to the outside of the bony labyrinth and is sandwiched between the dura mater (DM) and sigmoid sinus with the squamous part of the temporal bone (TB).

(F) A fine needle is used to cut around the endolymphatic sac (following the dotted yellow lines on panel D). 
(G) The preparation is carefully removed from the temporal bone by holding the tissue at the position shown by the asterisk $\left({ }^{*}\right.$, also shown in panel E) and peeling it up.

(H) An isolated whole endolymphatic sac with surrounding tissues.

(I) Schematic version of this isolated endolymphatic sac with surrounding tissues.

(J) Isolated endolymphatic sac without surrounding tissues.

Scale bars: $2 \mathrm{~mm}(B), 1 \mathrm{~mm}(\mathrm{~J})$. Please click here to view a larger version of this figure.
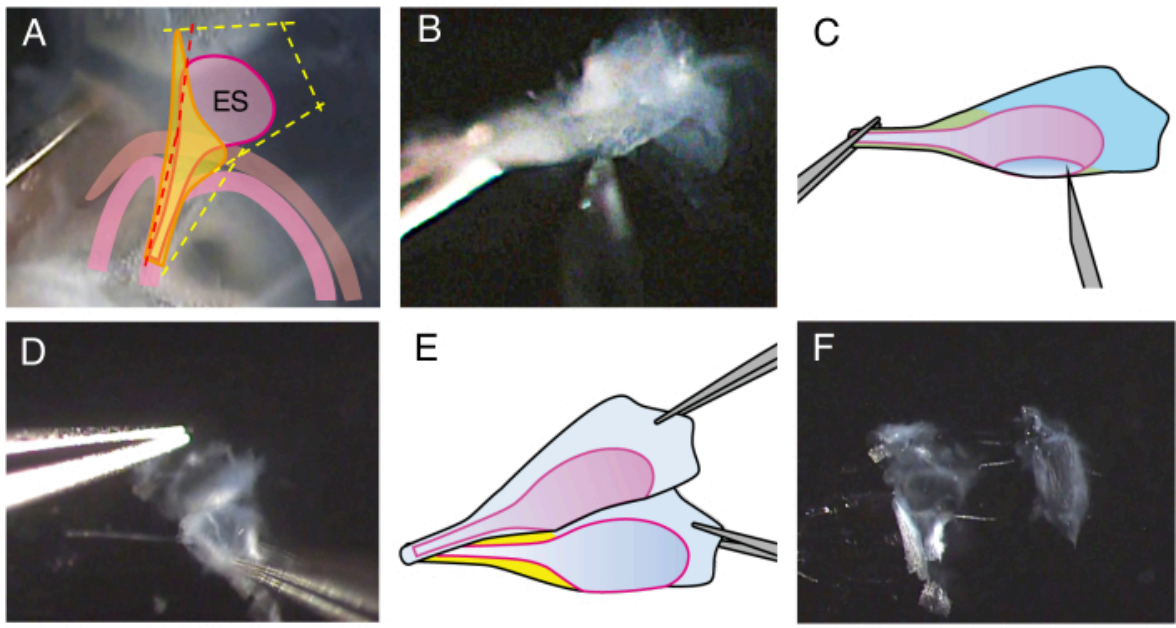

Figure 2. Dissection of the opened endolymphatic sac of a P5 wild type mouse.

(A) On step 2.4, the incision line on the vestibular aqueduct is positioned slightly anterior (red dotted line) to make an incision into the endolymphatic sac lumen.

(B) Hold the stem part of the preparation, insert a 27-gauge needle into the lumen, and move it to cut the endolymphatic sac into two sheets.

(C) Schematic illustration of panel B.

(D) Hold the edge of each sheet with forceps and separate them from each other.

(E) Schematic illustration of panel D.

(F) The endolymphatic sac is separated into two sheets, including the epithelium and the surrounding tissues. Please click here to view a larger version of this figure. 


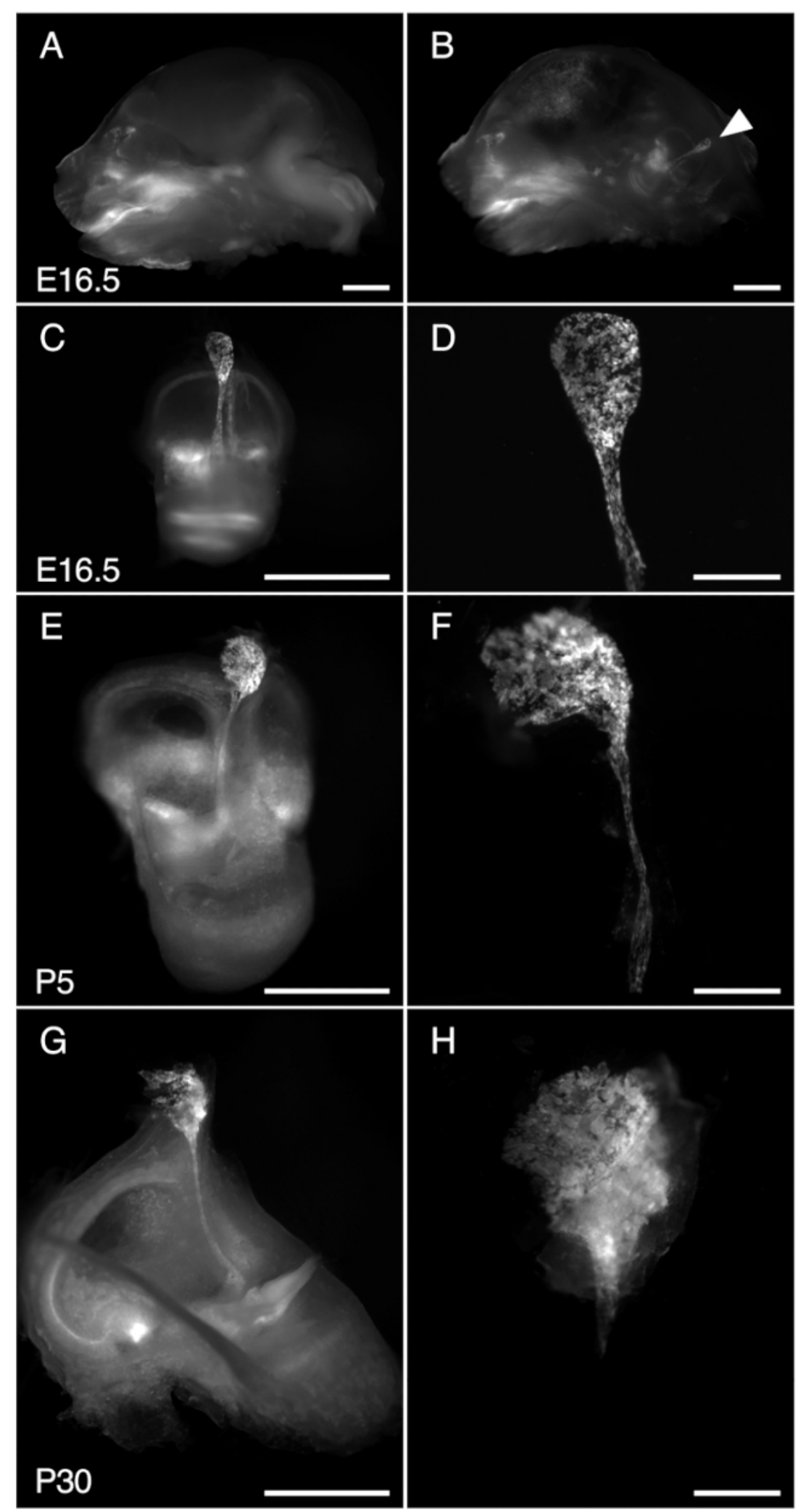


Figure 3. Dissection of $R 26^{L S L-R C L-t d T /+} ; \mathrm{Tg}(\mathrm{ATP} 6 \mathrm{~V} 1 \mathrm{~B} 1-\mathrm{Cre})^{1 \mathrm{Rnel} / \mathrm{Mn}}$ mouse endolymphatic sac at E16.5, P5, and P30. All images presented show tdTomato fluorescence obtained using a stereomicroscope with a $1 \mathrm{x}$ objective equipped for detection of tdTomato fluorescence.

(A, B) Mid-sagittal section of the skull of a E16.5 R26 ${ }^{L S L-R C L-t d T /+} ; \operatorname{Tg}(\mathrm{ATP} 6 \mathrm{~V} 1 \mathrm{~B} 1-\mathrm{Cre})^{1 \mathrm{Rnel} / \mathrm{Mn}}$ mouse before (A) and after (B) half-brain removal. tdTomato fluorescence outlines the position of the inner ear. The endolymphatic sac is readily visible even without dissection (arrowhead).

(C, E, G) Isolated inner ears from E16.5, P5 and P30 mice, respectively.

$(\mathrm{D}, \mathrm{F}$ and $\mathrm{H})$ Higher magnification images of the corresponding microdissected endolymphatic sacs and ducts. At P30, the endolymphatic duct is encapsulated in bone making it particularly difficult to isolate. Scale bars: $2 \mathrm{~mm}(\mathrm{~A}, \mathrm{~B}, \mathrm{C}, \mathrm{E}, \mathrm{G}), 500$ $\mu \mathrm{m}(\mathrm{D}, \mathrm{F}, \mathrm{H})$. Please click here to view a larger version of this figure.

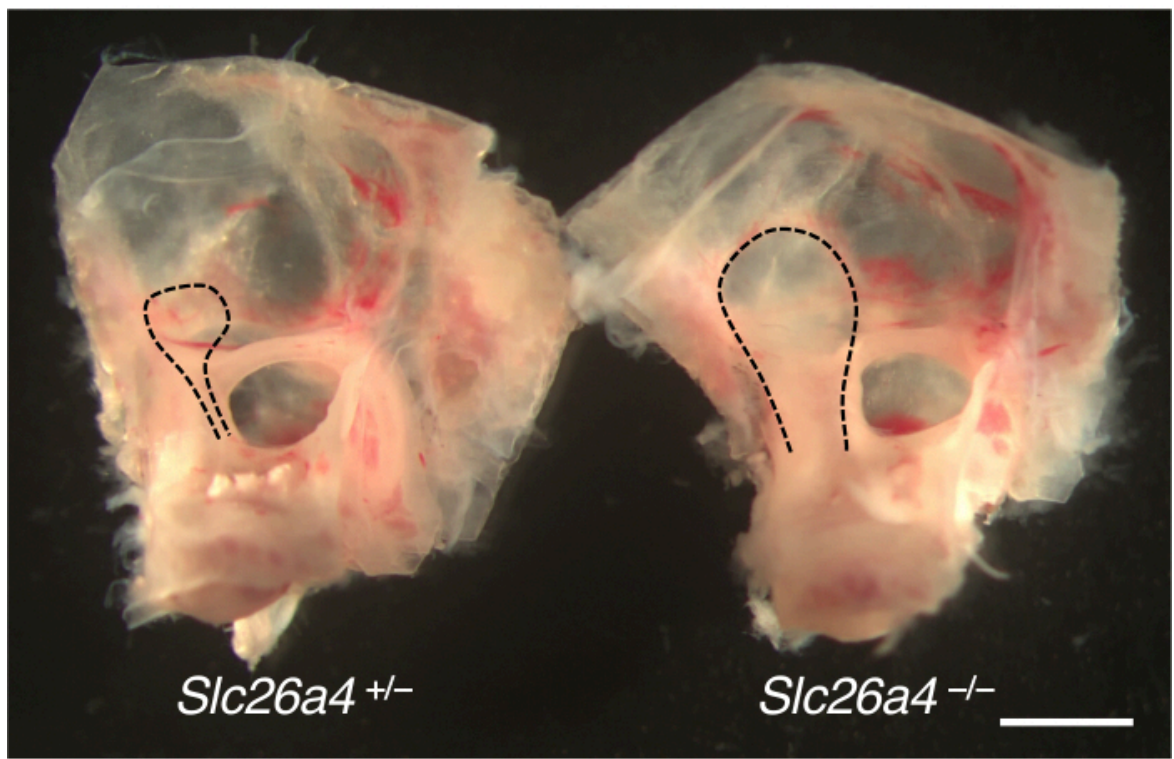

Figure 4. Gross anatomy of the otic capsule with an enlarged endolymphatic sac. Inner ears from S/c26a4 ${ }^{+/-}$(left) and Slc26a $4^{--}$(right) mouse littermates at P105. The vestibular aqueduct and endolymphatic sac and duct (black dotted lines) are enlarged in the S/c26a4 $4^{-/-}$mouse as compared to those in a S/c26a4 ${ }^{+/-}$mouse. Scale bar: $2 \mathrm{~mm}$. Please click here to view a larger version of this figure. 

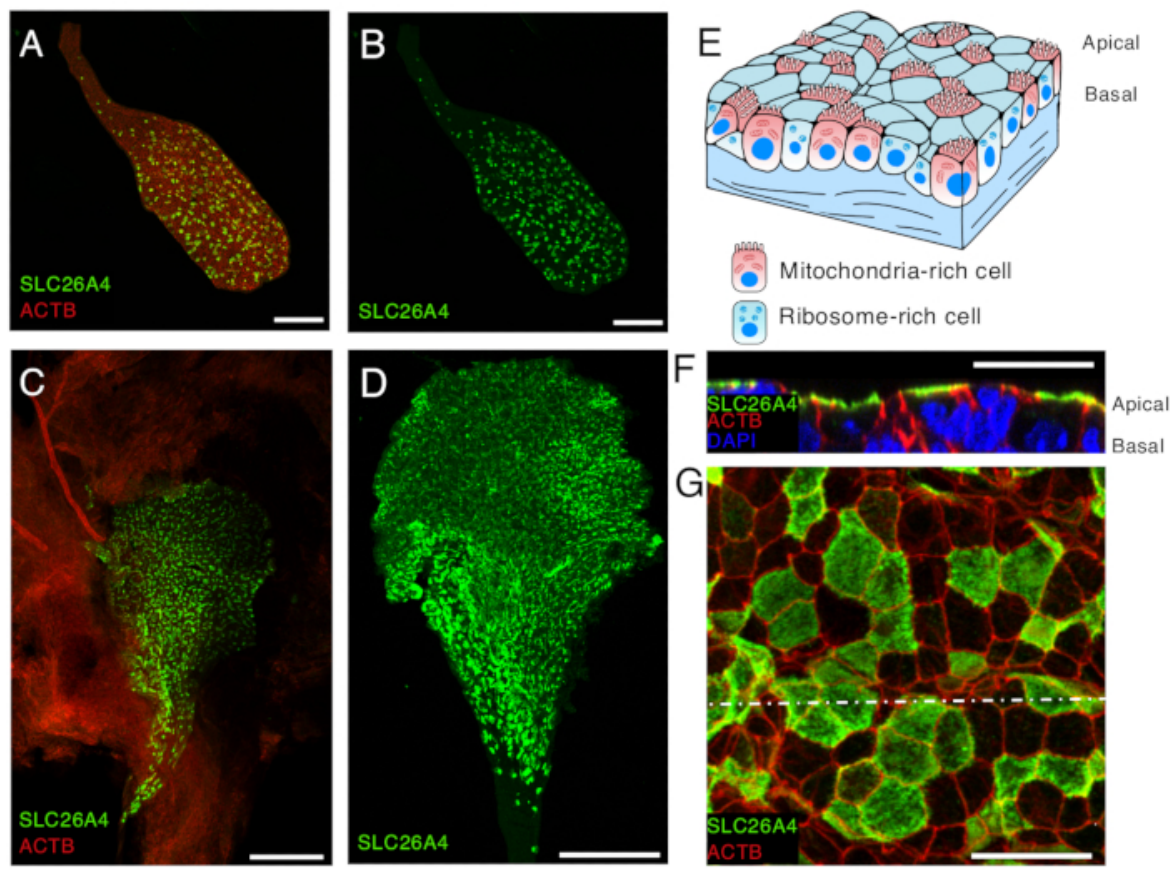

Figure 5. SLC26A4 expression of mitochondria-rich cells in the endolymphatic sac epithelium at E16.5 and P5.

(A, B) Isolated endolymphatic sac from E16.5 mouse labeled with anti-SLC26A4 antibody (green) and phalloidin which labels $\beta$-actin (ACTB, red).

(C) A low-magnification image of opened endolymphatic sac from P5 mouse labeled with an anti-SLC26A4 antibody (green). Phalloidin (ACTB, red) can be used to highlight the presence of the endolymphatic sac as well as the conjunctive tissue around it.

(D) Isolated endolymphatic sac labeled with an anti-SLC26A4 antibody (green).

(E) Schematic illustration of endolymphatic sac epithelium highlighting the presence of two cell types: the mitochondria-rich cells, which apical surface is covered with microvilli, and the ribosome-rich cells.

(F-G) High-magnification images of endolymphatic sac epithelium at P5, after the endolymphatic sac was opened and labeled with an anti-SLC26A4 antibody (green) and phalloidin (ACTB, red). A representative image at the apical membrane level is shown in G, and a reconstructed cross-section from z-stack at the level of the punctate white line is shown in $F$. The nucleus of the cells is labeled with DAPI (blue).

Scale bars: $100 \mu \mathrm{m}(\mathrm{A}, \mathrm{B}) ; 200 \mu \mathrm{m}(\mathrm{C}, \mathrm{D}) ; 20 \mu \mathrm{m}(\mathrm{F}, \mathrm{G})$. Please click here to view a larger version of this figure.

Supplementary Figure 1. Incident light and endolymphatic sac and duct can be readily visualized using corresponding tdTomato fluorescence images of a tdTomato fluorescence.

P5 $R 26^{\text {LSL-RCL-tdT/+}} ;$ Tg(ATP6V1B1-Cre) ${ }^{1 \text { Rnel/Mn }}$ mouse inner ear at three steps of the dissection. The 
(A-B) Region of the inner ear in the posterior part of an intact half skull of a P5 R26 ${ }^{L S L-R C L-t d T /+} ; \operatorname{Tg}(\mathrm{ATP} 6 \mathrm{~V} 1 \mathrm{~B} 1-\mathrm{Cre})^{1 \mathrm{Rnel} /}$ $\mathrm{Mn}$ mouse.

(C-D) Removal of the skin and part of the skull allows better visualization of the region of the endolymphatic sac.

(E, F) Isolated inner ear with the endolymphatic sac still associated with conjunctive tissue.

( $A, C, E)$ Images captured in incident light. (B, D, F) tdTomato fluorescence of the corresponding tissue. Scale bars: $2 \mathrm{~mm}$ (A-B), $1 \mathrm{~mm}(\mathrm{C}-\mathrm{F})$. Please click here to download this File.

\section{Supplementary Figure 2. Comparison of the fluorescence} of isolated endolymphatic sacs of $R 26^{L S L-R C L-}$ ${ }^{t d T /+} ; \mathrm{Tg}(\mathrm{ATP} 6 \mathrm{~V} 1 \mathrm{~B} 1-\mathrm{Cre})^{1 \mathrm{Rnel} / \mathrm{Mn}}$ and $R 26^{\text {LSL-RCL-tdT/+ }}$

littermate mice at E16.5 and P5. tdTomato fluorescence greatly facilitates the recognition of the endolymphatic sac and duct.

(A-C) Microdissected endolymphatic sacs of E16.5 R26 ${ }^{L S L-}$ ${ }^{R C L-t d T /+} ; \mathrm{Tg}(\mathrm{ATP} 6 \mathrm{~V} 1 \mathrm{~B} 1-\mathrm{Cre})^{1 \mathrm{Rnel} / \mathrm{Mn}}$ (A) and R26 ${ }^{L S L-R C L-}$ ${ }^{t d T /+}$ littermate mice $(\mathrm{B}, \mathrm{C})$.

(D-F) Microdissected endolymphatic sacs of P5 R26 $6^{L S L-R C L-}$ ${ }^{t d T /+} ; \operatorname{Tg}(\text { ATP6V1B1-Cre })^{1 \text { Rnel/Mn }}$ (D) and R26 $6^{L S L-R C L-t d T /+}$ littermate mice $(E, F)$.

(A, B, D, E) tdTomato fluorescence. (C, F) Incident light images corresponding to B and E. Scale bars: $500 \mu \mathrm{m}$. Please click here to download this File.

\section{Discussion}

Many researchers have used paraffin-embedded or frozen sections for morphological studies of the endolymphatic sac. The disadvantage of sectioned preparations is that it can be difficult to fully observe the complicated cell-to-cell contacts because the size and shape of each epithelial cell in the endolymphatic sac is extremely variable, forming a wrinkled and pseudostratified cell layer. Whole-mount immunostaining and z-stack imaging, described in this protocol, permit better visualization of the three-dimensional structure of the endolymphatic sac.

For successful microdissection, the critical point is to mentally visualize the entire outline of the endolymphatic sac, even if it is invisible in the wild type mouse. Observation of the fluorescent reporter shown in Figure $\mathbf{3}$ and in Supplementary Figure 1-2, can be helpful for recognizing localization and size of the endolymphatic sac in mice.

While this whole-mount dissection method was used for immunostaining in the above protocol, this technique can also be used to harvest endolymphatic sac tissue for gene expression analysis, such as RT-qPCR, microarray expression, and even single-cell RNA-seq. The results of single-cell RNA-seq analysis using tissue prepared with this protocol have been previously reported ${ }^{16}$. For gene expression analysis of endolymphatic sac epithelial cells, it is preferable to collect isolated epithelium so that there is no contamination with other adjacent tissues. Although the embryonic endolymphatic sac can be separated from the adjacent connective tissues, it is much harder to do at later ages. For specimens from older mice, incubation with collagenase/dispase for $5 \mathrm{~min}$ at $37^{\circ} \mathrm{C}$ is encouraged and facilitates the isolation of the endolymphatic sac epithelium.

One of the drawbacks of the whole-mount immunostaining described in this protocol is that the original three-dimensional structure may be affected by trauma during dissection or mounting of the preparation between glass slide and coverslip. Care is needed to avoid artefacts due to manipulation. 


\section{Disclosures}

The authors have nothing to disclose.

\section{Acknowledgments}

This research was supported in part by the Intramural Research Program of the NIH, NIDCD (NIH Intramural Research Funds Z01-DC000060 and ZIC DC000081 to the Advanced Imaging Core). We are grateful to Drs. Raoul D. Nelson and R. Lance Miller (University of Utah, Salt Lake City, UT) and Dr. Susan Wall (Emory University School of Medicine, Atlanta, GA) for sharing the Tg(ATP6V1B1Cre) mice and to the NIDCD animal facility staff for caring for our animals. We express our gratitude to Dr. Philine Wangeman for the beautiful illustration of the inner ear. We thank Drs. Thomas B. Friedman and Robert J. Morell for critically reviewing this manuscript.

\section{References}

1. Dror, A. A., Brownstein, Z., Avraham, K. B. Integration of human and mouse genetics reveals pendrin function in hearing and deafness. Cellular Physiology and Biochemistry. 28 (3), 535-544 (2011).

2. Reardon, W., CF, O. M., Trembath, R., Jan, H., Phelps, P. D. Enlarged vestibular aqueduct: a radiological marker of pendred syndrome, and mutation of the PDS gene. QJM. 93 (2), 99-104 (2000).

3. Griffith, A. J., Wangemann, P. Hearing loss associated with enlargement of the vestibular aqueduct: mechanistic insights from clinical phenotypes, genotypes, and mouse models. Hearing Research. 281 (1-2), 11-17 (2011).

4. Gurkov, R., Pyyko, I., Zou, J., Kentala, E. What is Meniere's disease? A contemporary re-evaluation of endolymphatic hydrops. Journal of Neurology. 263 Suppl 1 S71-81 (2016).

5. Eckhard, A. et al. Water channel proteins in the inner ear and their link to hearing impairment and deafness. Molecular Aspects of Medicine. 33 (5-6), 612-637 (2012).

6. Choi, B. Y. et al. Mouse model of enlarged vestibular aqueducts defines temporal requirement of Slc26a4 expression for hearing acquisition. Journal of Clinical Investigation. 121 (11), 4516-4525 (2011).

7. Miyashita, T. et al. Presence of FXYD6 in the endolymphatic sac epithelia. Neuroscience Letters. $\mathbf{5 1 3}$ (1), 47-50 (2012).

8. Royaux, I. E. et al. Localization and functional studies of pendrin in the mouse inner ear provide insight about the etiology of deafness in pendred syndrome. Journal of the Association for Research in Otolaryngology. 4 (3), 394-404 (2003).

9. Kim, H. M., Wangemann, P. Epithelial cell stretching and luminal acidification lead to a retarded development of stria vascularis and deafness in mice lacking pendrin. PLoS One. 6 (3), e17949 (2011).

10. Dahlmann, A., von During, M. The endolymphatic duct and sac of the rat: a histological, ultrastructural, and immunocytochemical investigation. Cell and Tissue Research. 282 (2), 277-289 (1995).

11. Everett, L. A. et al. Targeted disruption of mouse Pds provides insight about the inner-ear defects encountered in Pendred syndrome. Human Molecular Genetics. 10 (2), 153-161 (2001).

12. Miller, R. L. et al. The V-ATPase B1-subunit promoter drives expression of Cre recombinase in intercalated 
cells of the kidney. Kidney International. 75 (4), 435-439 (2009).

13. Madisen, L. et al. A robust and high-throughput Gre reporting and characterization system for the whole mouse brain. Nature Neuroscience. 13 (1), 133-140 (2010).

14. Wang, L., Jiang, H., Brigande, J. V. Gene transfer to the developing mouse inner ear by in vivo electroporation. Journal of Visualized Experiments. 64, e3643 (2012).

15. Currle, D. S., Mu, J. S., Kolski-Andreaco, A., Monuki, E. S. Culture of mouse neural stem cell precursors. Journal of Visualized Experiments. 2, e152 (2007).

16. Honda, K. et al. Molecular architecture underlying fluid absorption by the developing inner ear. Elife. 6, e26851 (2017).

Copyright $\odot 2021$ JoVE Creative Commons Attribution-NonCommercial-NoDerivs 3.0 Unsorted

jove.com

March $2021 \cdot 169 \cdot$ e62375 Page 13 of 13

License 